J Neurol Neurosurg Psychiatry 2005; 76(Suppl V):v15-v24. doi: 10.1136/jnnp.2005.081133

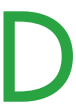
ementia is a clinical state characterised by a loss of function in at least two cognitive domains. When making a diagnosis of dementia, features to look for include memory impairment and at least one of the following: aphasia, apraxia, agnosia and/or disturbances in executive functioning. To be significant the impairments should be severe enough to cause problems with social and occupational functioning and the decline must have occurred from a previously higher level. It is important to exclude delirium when considering such a diagnosis.

When approaching the patient with a possible dementia, taking a careful history is paramount. Clues to the nature and aetiology of the disorder are often found following careful consultation with the patient and carer. A focused cognitive and physical examination is useful and the presence of specific features may aid in diagnosis. Certain investigations are mandatory and additional tests are recommended if the history and examination indicate particular aetiologies.

It is useful when assessing a patient with cognitive impairment in the clinic to consider the following straightforward questions:

- Is the patient demented?

- If so, does the loss of function conform to a characteristic pattern?

- Does the pattern of dementia conform to a particular pattern?

- What is the likely disease process responsible for the dementia?

An understanding of cognitive function and its anatomical correlates is necessary in order to ascertain which brain areas are affected. This, in turn, aids diagnosis. A discussion of the localisation of all cognitive processes is beyond the scope of this review. It is, however, particularly important to have an understanding of memory and its subdivisions, which is necessary to aid in differential diagnosis. We shall then illustrate how the history and examination, including bedside cognitive testing, are used in diagnosis.

\title{
TYPES OF MEMORY
}

When considering any memory disorder it is important to have an understanding of the main "types" of memory; otherwise erroneous use of the term "short term memory" may cause confusion. ${ }^{1}$ Memory can be thought of in terms of working memory, episodic memory (anterograde and retrograde), semantic memory, remote memory, and implicit memory (table 1). Classically, early Alzheimer's disease (AD) causes defects in anterograde episodic memory (for example, the ability to remember an address after five minutes or longer). ${ }^{2}$

The relevance of this is that aspects of memory are subserved by different structures. Certain disease processes have a tendency to start focally and progress in a typical anatomical pattern. They have, therefore, a largely predictable neuropsychological signature. For example, initially $\mathrm{AD}$ pathology tends to be perihippocampal, then spreads to temporo-parietal association cortex and latterly involves frontal lobes. This is mirrored by the initial cognitive deficit of anterograde episodic memory, progressing to attentional, semantic memory and visuo-perceptual impairment, with personality change occurring as a later feature.

TAKING A HISTORY AND THE EXAMINATION OF THE MEMORY IMPAIRED PATIENT It is vital to obtain a corroborating history from a relative or close friend in addition to the patient's account if they can provide one. The need to physically examine the patient, looking for signs pointing to a particular cause, is discussed below.

See end of article for authors' affiliations

Correspondence to: Dr John D W Greene, Southern General Hospital, Institute of Neurological Sciences, 1345 Govan Road, Glasgow G51 4TF, UK; john. greene@sgh.scot.nhs.uk

\section{History taking}

It is useful to interview the patient and the accompanying person separately. The absence of a concerned relative or friend at the appointment may lessen the likelihood of dementia in a patient complaining of memory problems. Interviewing the patient separately enables the cooperation and language skills to be assessed without them being masked by interruptions or assistance from a third party. It also allows an assessment into the degree of insight of the affected individual. Conversation with the patient may be as important as any formal cognitive assessment. The presence of word finding difficulties, paraphasic errors, and inappropriate behaviour is helpful in 


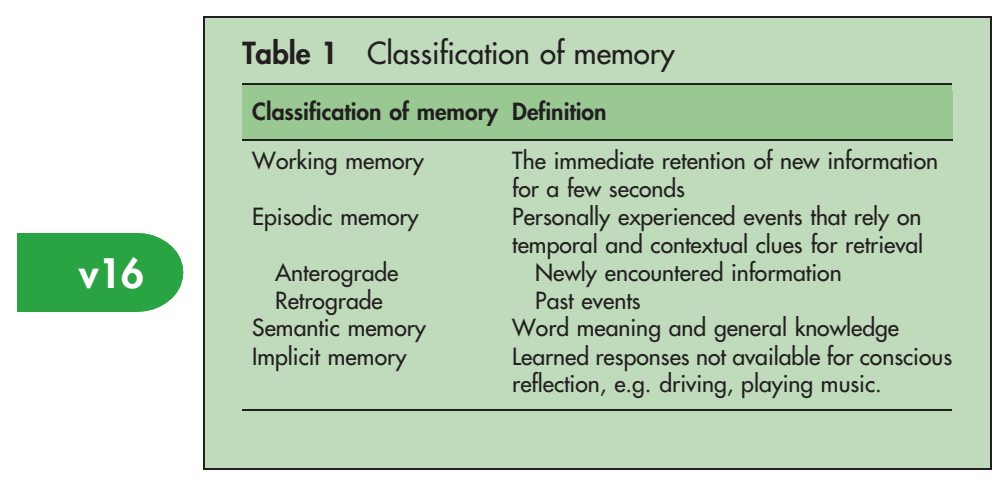

determining the likely cause of the dementia. Documentation of specific examples is a useful way of providing information that can be utilised by subsequent clinicians. Difficulties with specific aspects of memory are suggested by certain problems encountered in day to day activities. Anterograde memory deficiencies are suggested by the losing of objects, repetitive questioning, difficulty taking messages, an increasing reliance on lists, the failure to follow plots of films or television programmes, and getting lost (navigation). Semantic memory breakdown may manifest as a diminution of vocabulary with words being substituted by "thing". The meaning of unusual or infrequently used words may be lost. ${ }^{3}$

The level of alertness and cooperation during the interview should be assessed. If alertness is decreased, causes of this should be sought-for example, by a careful scrutiny of the drug history. Delirium as opposed to dementia should be considered if the patient appears poorly responsive.

Important features to note in the history include:

- symptoms at onset

- the tempo of evolution of symptoms

- the impact on work and family life

- issues of safety (for example, driving)

- a family history of dementia

- risk factors-for example. vascular

- past medical history.

The age of the patient is important as dementia in the young has a different aetiological profile than that seen in the elderly. AD, however, remains the most common dementia even in younger people, followed by vascular dementia and frontotemporal lobar degenerations. Genetic and metabolic disorders are more common in this age group and other diagnoses to consider include vasculitis, infections (for example, AIDS, tuberculous meningoencephalitis, Lyme disease, subacute sclerosing panencephalitis (SSPE)) and Creutzfeldt-Jakob disease (CJD). ${ }^{4}$

\section{Formal cognitive assessment}

A more detailed assessment of memory is necessary and performed by using several specific bedside cognitive tests. The role and method of using such tests has been covered in a previous supplement. ${ }^{5}$ During a thorough cognitive assessment it is useful to examine the following:

- Orientation -in time and place

- Attention-for example, serial sevens, months of the year or WORLD backwards

- Memory-for example, address recall, name of prime minister, etc

- Language-for example, naming of items, reading, writing, comprehension, repetition

- Executive function-for example, letter and category fluency

- Praxis-for example, alternating hand movements, imitation of gestures
- Visuospatial function-for example, drawing a clock face, overlapping pentagons.

\section{Rating scales}

The widely used mini mental state examination (MMSE) provides useful information in grading established dementia but does have limitations, particularly in detecting early disease. It contains a crude test of delayed recall, with only three items being employed and not enough time allowed between registration and recall. It lacks a timed test to detect problems with verbal fluency. The language items are also very easy, with all but significantly aphasic patients tending to perform at ceiling on these items. ${ }^{6}$

The Addenbrooke's cognitive assessment (ACE) has been developed to address the deficiencies of the MMSE. ${ }^{7}$ It also has the advantage of being brief enough to allow a clinician to use it within the time constraints of a new patient appointment. ${ }^{7}$ It should be noted that even the ACE is no match for formal neuropsychology assessment. Such services, are, however, patchy, and in some services are non-existent, so the clinician must remain competent at assessing cognition.

\section{THE FOCUSED EXAMINATION OF THE PATIENT WITH DEMENTIA Neurological system}

Aside from the mental state examination and specific tests of cognitive function it is important to examine the neurological system in any patient with possible cognitive impairment. Neurological examination is, however, often normal in the early stages of many neurodegenerative dementias and specific abnormalities may point to rarer or potentially treatable causes of dementia (table 2).

It is important to assess the patient at rest for any involuntary movements, including chorea, tremor, dystonia, and myoclonus (which may be spontaneous or stimulus sensitive). The muscles should be observed for fasciculations. The presence or absence of primitive reflexes (frontal release signs) should be determined. An ocular examination should involve careful assessment of visual acuity, pupillary responses, eye movements, optic discs, and visual fields. Assessment of speech and swallowing may reveal the presence of bulbar features. Examination for pyramidal or extrapyramidal signs is important and gait should be assessed wherever possible. Ataxia is unusual in $A D$, dementia with Lewy bodies, and frontotemporal dementia; its presence should raise the possibility of a different cause. The presence or absence of apraxia should be assessed by asking the patient to perform alternating hand movements or copy gestures. A peripheral neuropathy may be present and when cooperation allows signs of this should be sought. The significance of these findings in relation to specific potential diagnoses is outlined in table 2.

\section{Examination of other systems}

Examination of other systems is also useful in looking for evidence of multisystem disease. In addition to the neurological examination, patients should be assessed for signs of immunocompromise (predisposing to opportunistic infections such as progressive multifocal leucoencephalopathy (PML), toxoplasmosis or primary cerebral lymphoma possibly indicating HIV/AIDS). Features of systemic disease may indicate an underlying neoplasm, vasculitis, infection, or a metabolic disorder. Uveitis may indicate sarcoidosis, Behçet's 
Table 2 Abnormal neurological signs and their significance in dementia

\begin{tabular}{|c|c|}
\hline Physical sign & Seen in \\
\hline Ataxia & $\begin{array}{l}\text { Paraneoplastic disease, cerebellar tumour, Whipple's disease, Creutzfeldt-Jakob disease (CID), AIDS dementia complex, } \\
\text { spinocerebellar ataxia (SCA), Wernicke-Korsakoff syndrome, Hallervorden-Spatz, ornithine transcarbamylase deficiency, } \\
\text { Niemann-Pick disease, mitochondrial disorders, adrenoleucodystrophy, neurodegeneration with brain iron accumulation } \\
\text { (NBIA), lead poisoning }\end{array}$ \\
\hline Involuntary movements & $\begin{array}{l}\text { Huntington's disease (HD), inherited metabolic disorders including Wilson's disease, CJD, corticobasal degeneration (CBD), } \\
\text { systemic lupus erythematosis, Whipple's disease, Hallervorden Spatz, Lesch-Nyhan }\end{array}$ \\
\hline Myoclonus & $\begin{array}{l}\text { Post-anoxia, CJD, Alzheimer's disease (AD), subacute sclerosing panencephalitis (SSPE), myoclonic epilepsies, Hashimoto's } \\
\text { encephalopathy, dementia with Lewy bodies, CBD }\end{array}$ \\
\hline Extrapyramidal signs & $\begin{array}{l}\text { Dementia with Lewy bodies, Parkinson's disease, progressive supranuclear palsy (PSP), vascular dementia, frontotemporal } \\
\text { dementia (FTD), CJD, Wilson's disease, HD, dentato-rubro-pallido-luysian atrophy (DRPLA), neuroacanthocytosis, cerebral } \\
\text { autosomal dominant arteriopathy with subcortical infarcts and leucoencephalopathy (CADASIL), Niemann-Pick, mitochondrial } \\
\text { disorders, NBIA }\end{array}$ \\
\hline Pyramidal signs & $\begin{array}{l}\text { Motor neuron disease, CJD, LCBD, B12 deficiency, multiple sclerosis (MS), SCA, multi system atrophy, hydrocephalus, AD, } \\
\text { Hallervorden Spatz, CADASIL, mitochondrial disorders, adrenoleucodystrophy, FTD }\end{array}$ \\
\hline Optic disc pallor & MS, B12 deficiency \\
\hline Papilloedema & Tumour, subdural haematoma, hydrocephalus \\
\hline Cortical blindness & Vascular disease, AD, CJD \\
\hline Anosmia & Subfrontal meningioma, head injury, $A D, P D, H D$ \\
\hline Abnormal eye movements & $\begin{array}{l}\text { Progressive supranuclear palsy, Wernicke-Korsakoff, Whipple's disease, CBD, mitochondrial cytopathies, cerebellar tumours, } \\
\text { causes of raised intracranial pressure, CJD, mitochondrial disorders, HD, Niemann Pick type C }\end{array}$ \\
\hline Other cranial nerve signs & Sarcoidosis, tumours, neoplasia, tuberculous meningitis \\
\hline Alien hand & CBD \\
\hline Visual field defect & Tumour, vascular disease, CJD \\
\hline $\begin{array}{l}\text { Pupillary abnormalities (Argyll } \\
\text { Robertson pupil) }\end{array}$ & Neurosyphilis \\
\hline Peripheral neuropathy & $\begin{array}{l}\text { Vitamin B12 deficiency, paraneoplastic disorders, neuroacanthocytosis, spinocerebellar ataxia, Hallervorden Spatz, } \\
\text { adrenoleucodystrophy, NBIA, lead poisoning, systemic lupus erythematosus (SLE) }\end{array}$ \\
\hline Early onset incontinence & Tumour, hydrocephalus, PSP \\
\hline Bulbar features & Frontal dementia (motor neuron disease) \\
\hline Fasciculations & Frontal dementia (motor neuron disease), rarely CJD \\
\hline Seizures & $\begin{array}{l}\text { Vasculitis, neoplasia, primary angiitis of the nervous system, limbic encephalitis, AIDS dementia complex, neurosyphilis, SSPE, } \\
\text { Hashimoto's encephalopathy }\end{array}$ \\
\hline Grimacing facial expression & Wilson's disease \\
\hline
\end{tabular}

disease, or multiple sclerosis (MS). The presence of cardiac disease, hypertension, or a previous transient ischaemic attack or stroke may suggest cerebrovascular disease.

Armed with the above theoretical knowledge regarding memory and its subdivisions along with how to elicit information from history taking and examination, we can now return to trying to achieve a diagnosis in a patient with possible dementia.

\section{IS IT DEMENTIA?}

\section{Conditions mimicking dementia}

The first and most important question to be asked when assessing the memory impaired patient is "Is this dementia?". Conditions mimicking dementia are considered under the term "pseudodementia" and often relate to affective disorders that represent treatable psychiatric pathology, such as anxiety or depression.

Table 3 Clinical features of delirium versus dementia

\begin{tabular}{|c|c|c|}
\hline Feature & Delirium & Dementia \\
\hline Progression & $\begin{array}{l}\text { Fluctuating with lucid } \\
\text { intervals, often worse at } \\
\text { night }\end{array}$ & $\begin{array}{l}\text { Fairly consistent over the } \\
\text { course of a day }\end{array}$ \\
\hline Consciousness & Altered & Clear \\
\hline Attention & $\begin{array}{l}\text { Impaired with pronounced } \\
\text { distractibility }\end{array}$ & Relatively normal \\
\hline Memory & Impaired & Impaired \\
\hline Thinking & Disorganised, delusional & Paucity of thought \\
\hline $\begin{array}{l}\text { Sleep-wake } \\
\text { cycle }\end{array}$ & Disrupted & Often normal \\
\hline Perception & $\begin{array}{l}\text { Hallucinations and illusions } \\
\text { common, often visual }\end{array}$ & $\begin{array}{l}\text { Hallucinations generally } \\
\text { absent in early stages }\end{array}$ \\
\hline
\end{tabular}

Certain features may point to an early pseudodementia rather than a dementia, but often it is the clinical assessment over time that enables a distinction between the two to be made. Biological features of depression should be enquired about in all cases (anorexia, weight loss, sleep disturbance, and motor retardation) although none of these are exclusive to pseudodementia. Emotional blunting, loss of interest, pessimism, guilt, and negative ruminations may be present. An identifiable emotional precipitant may be present in pseudodementia, along with a relatively abrupt onset and lack of progression. Some organic dementias, however, present with anxiety and depression and may be mistaken initially for a pure psychiatric illness.

Delirium - that is, an acute confusional state-may also be mistaken for dementia. Delirium is characterised by an abrupt onset of pronounced attentional abnormalities with disordered perception and memory. There is often significant variation in cognitive performance and behaviour. Table 3 illustrates some of the clinical differences observed between delirium and dementia.

IF IT IS DEMENTIA, WHAT SORT OF DEMENTIA IS IT? The whole brain is not affected equally in dementia. Psychological processes are organised into specific brain areas and therefore different diseases reflect the distribution of pathology within the anterior, medial, and posterior cortex by producing distinct neuropsychological syndromes.

\section{Cortical and subcortical dementias}

Deficits in certain areas, especially relatively early in the disease, may point to a specific dementia. One of the more widely used categorisations of dementia is into cortical (predominantly involving the cerebral cortex) and subcortical 
Table 4 A comparison of cortical and subcortical dementia according to neuropsychological profile

\begin{tabular}{|c|c|c|}
\hline Characteristic & Cortical & Subcortical \\
\hline $\begin{array}{l}\text { Speed of cognitive } \\
\text { processing }\end{array}$ & Normal & Slowed \\
\hline $\begin{array}{l}\text { Planning, problem } \\
\text { solving, initiative (frontal } \\
\text { "executive" abilities) }\end{array}$ & $\begin{array}{l}\text { Preserved in early } \\
\text { stages }\end{array}$ & Impaired from onset \\
\hline Personality & $\begin{array}{l}\text { Intact until late, unless } \\
\text { frontal type }\end{array}$ & Apathetic, withdrawn \\
\hline Memory & Severely amnesic & Forgetful \\
\hline Language & Aphasia & $\begin{array}{l}\text { Normal except for } \\
\text { dysarthria and } \\
\text { reduced output }\end{array}$ \\
\hline $\begin{array}{l}\text { Visuospatial and } \\
\text { perceptual difficulties }\end{array}$ & Impaired & Impaired \\
\hline Mood & $\begin{array}{l}\text { Depression not } \\
\text { uncommon in early } \\
\text { Alzheimer's disease }\end{array}$ & Depression common \\
\hline Agnosia/prosopagnosia & Often present & Not usually seen \\
\hline
\end{tabular}

forms (primarily affecting the basal ganglia, thalamus, and deep white matter) (table 4). Examples of cortical dementias include $\mathrm{AD}$ and CJD. The clinical manifestations of cortical dementias include agnosia, spatial disorientation, language problems, apraxia, amnesia, and problems with visuospatial functioning depending on the location of the pathology.

Examples of subcortical dementias include Parkinson's disease, Huntington's disease, vascular dementia, progressive supranuclear palsy, Wilson's disease, and AIDS dementia complex. Patients with a subcortical dementia show slowness and rigidity of thinking (bradyphrenia) often with perseveration. Although forgetful, they do not have a severe amnesia. There is difficulty in planning and sequencing of events and the pattern of cognitive impairment may be similar to that seen in frontal lobe dysfunction.

Some disorders display signs of both a cortical and subcortical dysfunction relatively early in the disease. Examples of cortico-subcortical conditions include cortical dementia with Lewy Bodies (DLB) and corticobasal degeneration (CBD).

\section{WHICH DEMENTIA IS IT?}

After detailed history taking and examination, the clinician may be confident which disease process is responsible for the symptoms. Often, however, the clinical picture will not be sufficiently distinctive to allow the clinician to be certain which type of dementia is responsible. For this reason-as
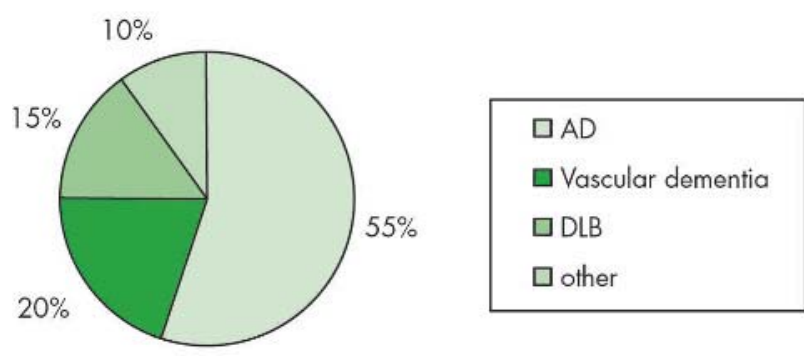

Figure 1 Approximate relative frequencies of the dementias. AD, Alzheimer's disease; DLB, dementia with Lewy bodies. well as the importance of never missing a treatable cause of dementia-it is usually appropriate to arrange investigations.

\section{INVESTIGATIONS}

Recommended investigations in all patients with dementia include full blood count, erythrocyte sedimentation rate, urea and electrolytes, liver function tests, vitamin B12, red cell folate, thyroid function, chest $x$ ray, and computed tomographic (CT) brain scan. In some circumstances, it may be considered appropriate to perform immunological tests for vasculitis, serum angiotensin converting enzyme, HIV testing, paraneoplastic antibodies, and screening for inborn errors of metabolism. Additional investigations are recommended in specific circumstances and include brain magnetic resonance imaging (MRI), functional imaging (SPECT), cerebrospinal fluid analysis, and electroencephalography. Further specialised investigations to be considered include a slit lamp examination (looking for the Kaiser-Fleischer rings of Wilson's disease), cardiac screening for emboli, and further specific genetic screening for individual disorders. Cerebral biopsy should be considered if a treatable cause is thought possible (for example, a cerebral vasculitis) in the absence of an alternative diagnosis. The role of laboratory and imaging investigations is examined by Weisje von der Flier et al in this supplement ( $\mathrm{p}$ v45).

\section{TYPES OF DEMENTIA}

\section{Alzheimer's disease (AD)}

$\mathrm{AD}$ is the most common cause of dementia, accounting for approximately two thirds of all cases (fig 1). AD is associated with age and Down syndrome. There also exists familial AD, associated with mutations of APP (amyloid $\beta$ protein precursor), PS (presenilin)-1, and PS-2. The disease results from the accumulation of abnormal $\beta$ amyloid leading to secondary neuronal injury and accumulation of tau in neurofibrillary tangles.

$\mathrm{AD}$ does not exhibit a global decline from onset but rather a relatively predictable pattern through various stages. By far the most common presentation is with amnesia, in particular a failure of anterograde episodic memory. Delayed recall (for example, a name and address after five minutes) is the most sensitive measure of early AD. Typically patients with AD perform well on test of working memory, including digit span. ${ }^{2}$ Although focal features have been reported at onset in $\mathrm{AD}$ these represent the very small minority. Depression is relatively common early in the disease and this may cause problems with diagnosis.

A progressive disturbance of semantic memory is seen as the disease advances and verbal fluency becomes impaired. Category fluency is more severely affected than letter based verbal fluency. Remote memory for famous faces and events is impaired and shows a gentle temporal gradient (that is, more distant memories are relatively well preserved compared with more recently acquired ones). In the middle stages of the disease it is common to find visual and perceptual difficulties emerging. Ideomotor apraxia occurs rendering tasks such as dressing and eating difficult. Language skills decline as the illness progresses and paucity of speech is evident. Comprehension is impaired and reading, writing, and calculation all become affected.

In notable contrast to frontotemporal lobar degeneration (FTD), basic aspects of personality, demeanour, social interaction, and behaviour are strikingly preserved well into the disease. Most patients retain at least partial insight for some time. 


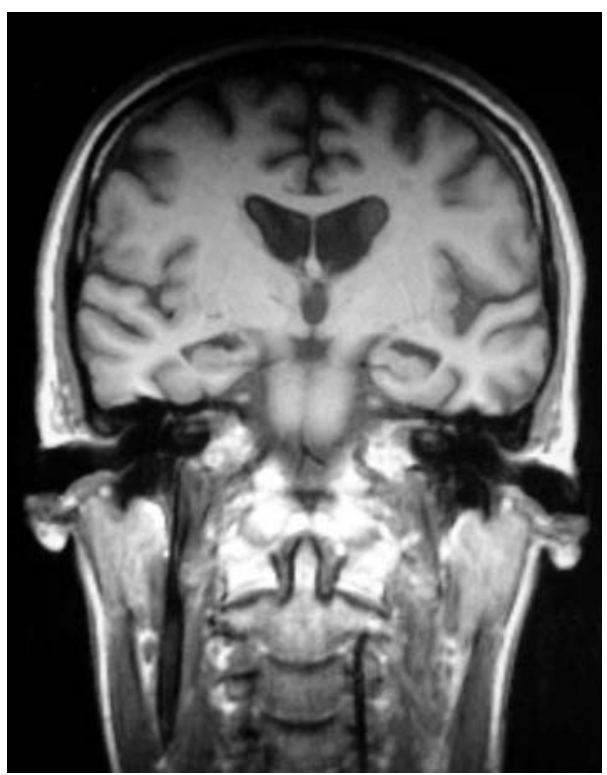

Figure 2 Magnetic resonance imaging (MRI) in Alzheimer's disease: bilateral hippocampal atrophy with generalised cortical atrophy.

Clinical diagnostic criteria have been developed that may be about $80 \%$ sensitive (NINCDS-ADRDA) although these are not widely used in the clinic in the authors' experience.

In $\mathrm{AD}$, as the disease progresses akinesia, rigidity, and myoclonus may all develop, reflecting the more widespread involvement of cortical and subcortical structures. Both extrapyramidal and pyramidal signs may be observed in later stages and myoclonus seen in a minority. By this stage cognition is often severely impaired with a disintegration of personality and incontinence. Death often occurs when immobility or poor nutrition predispose to bronchopneumonia.

It is worth stressing that it is not uncommon for a combination of pathologies to exist-that is, $\mathrm{AD}$ and cerebrovascular disease. Figures 2 and 3 show the appearances that may be seen on MRI and SPECT scanning in AD.

\section{Creutzfeldt-Jakob disease}

CJD is an invariably fatal prion disease of humans which has been described in sporadic, genetic, variant, and iatrogenic forms. All forms of CJD are characterised histologically by neuronal loss, gliosis, and spongiform change along with the deposition of an abnormal proteinaceous particle (the prion). Sporadic CJD is the most common form of CJD and exists worldwide (or at least wherever surveillance systems are in place) at an incidence of approximately one case per one million of the population per year. Sporadic CJD is characterised by an extremely rapidly progressive dementia with death occurring in the majority by six months after onset. Myoclonus is a commonly observed sign with other hard neurological signs (such as pyramidal features or ataxia) often observed. Variant CJD emerged in the UK in 1996 and is commonly regarded as representing the transmission of bovine spongiform encephalopathy to humans probably after the consumption of contaminated beef products. Affected individuals are typically younger than those with sporadic CJD (the mean age at onset is 28 years). Early sensory symptoms and psychiatric features are hallmarks of variant CJD. CJD may also be inherited in an autosomal dominant manner if there is an associated mutation in the gene encoding for normal prion protein. CJD has been reported iatrogenically as a result of human growth hormone injections, neurosurgery, and corneal grafting.

Cerebral MRI is useful in variant CJD where high signal bilaterally in the posterior thalamus (the "pulvinar sign") is frequently seen (fig 4), particularly on FLAIR (fluid attenuated inversion recovery) sequences. In sporadic CJD high signal in the basal ganglia structures may be observed although this is less specific than the pulvinar sign. Cerebrospinal fluid analysis for the 14-3-3 protein shows a high sensitivity and specificity in sporadic CJD if the patient group is carefully selected. The electroencephalogram (EEG) has been reported as showing periodic, sharp wave complexes in up to two thirds of patients with sporadic CJD.

\section{SUBCORTICAL DEMENTIAS}

\section{Progressive supranuclear palsy (PSP)}

This disorder characteristically presents with a parkinsonian syndrome, with cognitive dysfunction being an early and consistent feature. There is bradykinesia, extrapyramidal rigidity, and a tendency to falls experienced usually within a year of onset. Age at onset is typically over 40 years and progression is gradual. A vertical gaze supranuclear palsy is characteristic, particularly if downward saccades are impaired. Cognitive deficits consist of problems with executive function with cognitive slowing and difficulty planning and sequencing events. There is often a reduction in verbal output reflected in a poor performance in tests of verbal fluency. Patients show defective learning with impaired recall.

\section{Idiopathic Parkinson's disease (IPD)}

Recent studies suggest that up to three quarters of older patients with idiopathic Parkinson's disease go on to develop dementia. Risk factors for the development of dementia in IPD include older age, bradykinesia, akinetic-rigid IPD, depression, early visual hallucinations, and a bilateral onset of IPD. The major cognitive dysfunctions occur in the areas of executive function and memory. In common with other subcortical dementias language abilities are relatively spared in IPD.

\section{Huntington's disease}

Huntington's disease is a fully penetrant, autosomal dominant condition caused by a CAG repeat expansion in exon 1 of ITl5 gene encoding the Huntingtin protein. It is the most common genetic disorder to cause dementia. Onset of disease is most common in late middle age. There is often a prior history of a mood disorder and the disease is characterised by a slow deterioration of intellectual function along with personality change. Minor motor abnormalities are observed initially-for example, fidgety movements of the hands and feet during times of stress and general restlessness. As the disease progresses extrapyramidal signs are observed, often as chorea with dystonia, parkinsonism, and bradykinesia. Attentional and frontal executive dysfunction is seen early in the disease. Memory impairment is seen and the deficit is primarily caused by impaired retrieval of new information. Depression is common. ${ }^{4}$ Young patients may present atypically with an extrapyramidal syndrome, the so-called Westphal variant. 


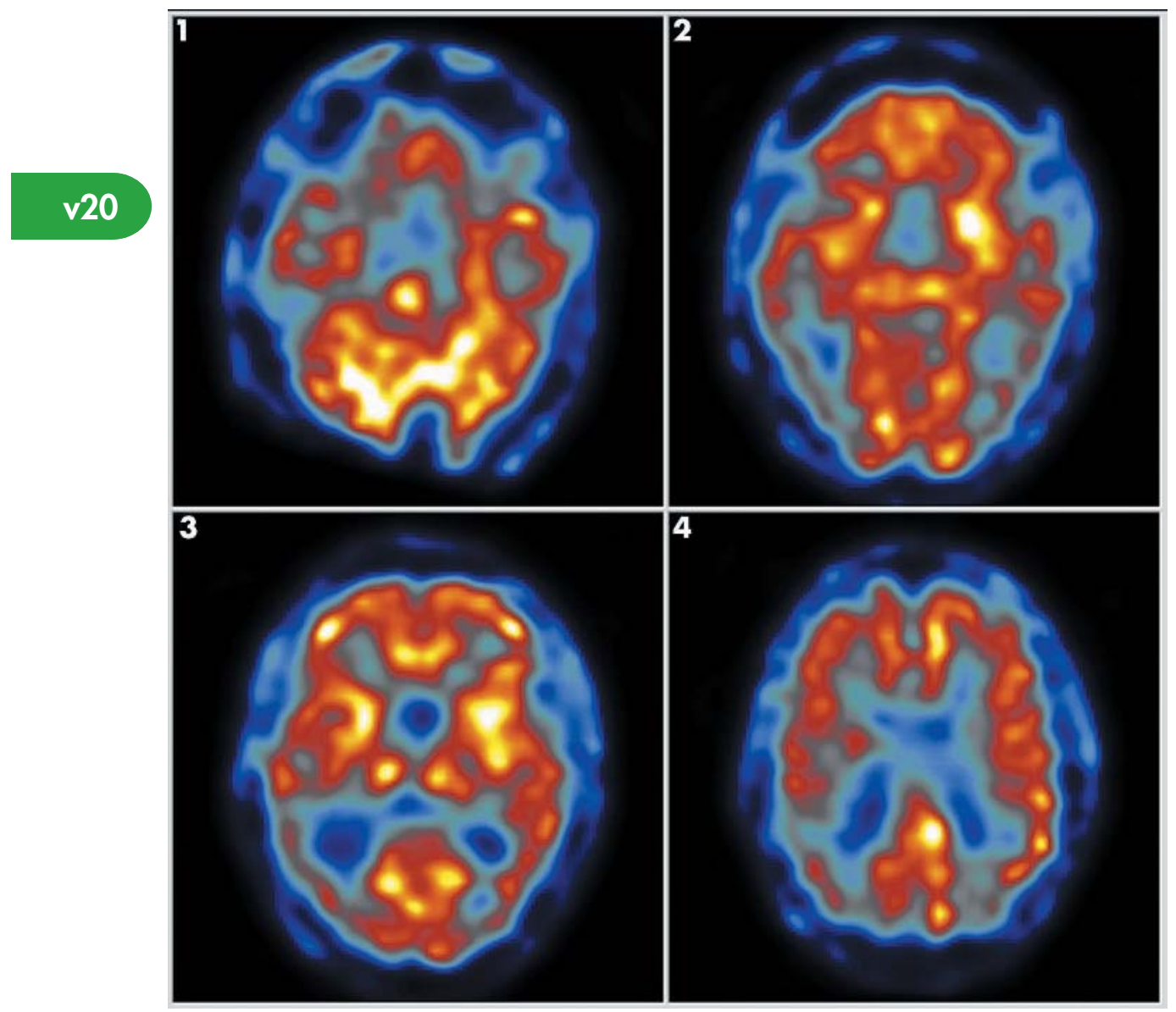

Figure 3 Typical posterior hypoperfusion seen on SPECT (single photon emission computed tomography) in Alzheimer's disease.

\section{MIXED CORTICAL-SUBCORTICAL DEMENTIAS Frontotemporal dementia (FTD)}

Frontotemporal degeneration is associated with a focal degeneration of the frontal and temporal lobes. Several variants exist within this group including a dementia of frontal type, progressive aphasia, and semantic dementia.

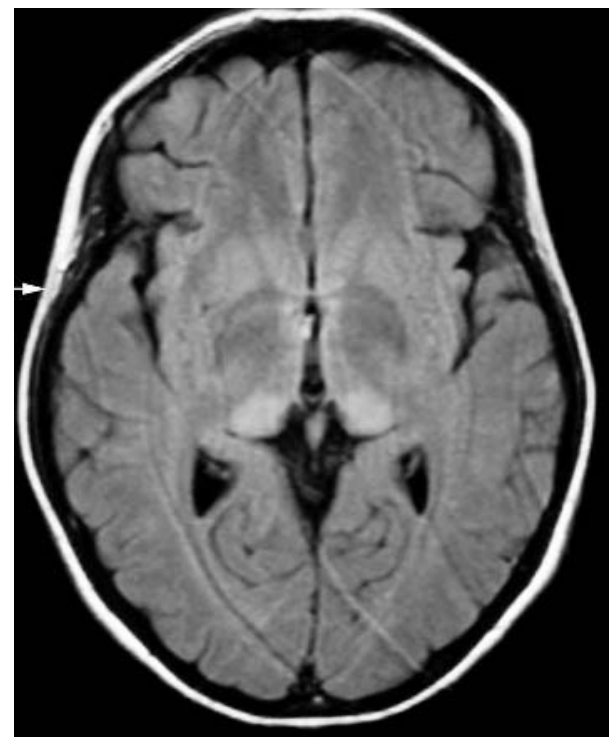

Figure 4 MRI showing the pulvinar sign (high signal in the posterior thalamus) seen in variant Creutzfeldt-Jakob disease (CJD).
The aetiology of the condition is unknown although it may be seen after the development of motor neuron disease when the neuronopathy is of the amyotrophic form displaying bulbar palsy, weakness, wasting, and fasciculations.

The pathological changes seen in FTD are varied. Many cases are associated with tau inclusions but mild spongiform change with neuronal loss and gliosis may occur in the absence of inclusions. The clinical phenotype reflects the anatomical distribution of the pathology, rather than the particular pathological process.

\section{The frontal variant of FTD}

In contrast to patients with $\mathrm{AD}$, those with frontal dementia often remain utterly unaware of the changes wrought to their personality. The initial presentation may be subtle but is characterised by personality change, emotional problems, and behavioural disturbance. Patients may appear apathetic, withdrawn, inappropriately jocular, socially disinhibited, facetious, or unmotivated. There is a reduced capacity to demonstrate appropriate emotional responses such as happiness, fear, and surprise. Sympathy, empathy, and embarrassment are often lacking and may be replaced by impulsivity and carelessness.

The presentation is quite distinct from that seen in $\mathrm{AD}$. Memory is typically unaffected early in the course of the disease with problems largely secondary to poor concentration and usually relating to difficulties with working (immediate) memory. The severe amnesic presentation of $\mathrm{AD}$ is not the pattern seen here. 


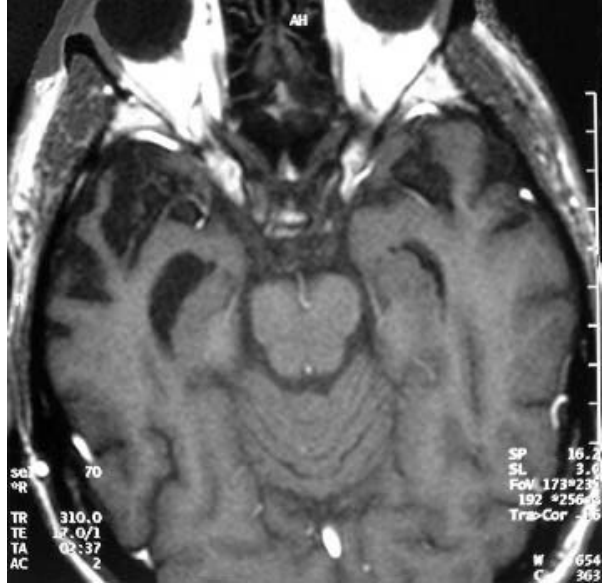

Figure 5 Bilateral anterior temporal lobe atrophy in frontotemporal dementia.

Impairment in planning and organising of complex activities is almost invariably observed in FTD. This reflects deficits in sustained attention, goal setting, and flexibility. Such difficulties may manifest through problems functioning at work or the inability to manage finances. When confronted with their failures patients appear disinterested and unperturbed. Mental rigidity and an inability to appreciate the more subtle aspects of language, such as irony, are common. Verbal perseveration and echolalia frequently occur. Speech is factually empty and reduced in quantity and has been described as "concrete". In advanced cases comprehension becomes impaired and muteness ensues.

Stereotyped and perseverative behaviours may develop. Deterioration in self care with neglect of washing and grooming is often reported. Many patients develop a sweet tooth and exhibit hyperorality. Sparing of the posterior cortices means that visuospatial problems are absent until the terminal stages. Neurological signs are minimal and consist of primitive reflexes, with akinesia and rigidity observed in the terminal stages. ${ }^{8}$

\section{Semantic dementia: progressive fluent aphasia}

This variant of FTD reflects selective atrophy of the left anterior temporal lobe (fig 5). Patients display increasingly empty, circumlocutory speech reflecting the profound loss of semantic knowledge. Presenting complaints may relate to forgetting of names of things with an unawareness of the parallel decline in word comprehension. The fluent dysphasia observed in such patients is coupled with a severe anomia, reduced vocabulary, and a pronounced impairment of single word comprehension. Patients exhibit a surface dyslexiathat is, an inability to read words with irregular spelling such as dough, pint, or island.

Such patients are often erroneously described as having a poor memory or being confused, as the language disorder makes it impossible for them to process verbal material. Assessment of memory in aphasic patients should be based on practical day-to-day activities and on tests of non-verbal memory. Figure 6 show bilateral frontotemporal hypoperfusion in a SPECT scan seen in frontotemporal dementia.

\section{Progressive non-fluent aphasia}

This variant of FTD reflects focal left perisylvian atrophy. Here, a progressive decline in language output occurs with a relative absence of other psychological deficits. Speech is non-fluent, effortful, and lacking in prosody. Articulation is disturbed, word finding pauses occur, and syntactic errors are prominent. The communication difficulties are evident to both the patient and the observer. Repetition and reading aloud are impaired and there is pronounced anomia. Patients have difficulties reciting the days of the week or similar well rehearsed series. With disease progression speech becomes unintelligible. Comprehension is, by contrast, relatively preserved.

\section{Vascular dementia}

The term "vascular dementia" is hampered by lack of agreement regarding definition. It comprises several different entities, such as multi-infarct disease, large cortical infarcts, and diffuse small vessel ischaemia.

The clinical picture in vascular dementia depends on the site and number of the infarcts. Diagnosis depends on:

the clinical picture

- brain imaging findings

- the presence of predisposing factors.

There is commonly an accumulation of neurological and psychological deficits. There may be multiple cortical infarcts causing a "step-wise" deterioration in function. Dysarthria, dysphagia, rigidity, visuospatial deficits, ataxia, and pyramidal or extrapyramidal signs may occur depending on the site of the pathology. Alternatively, subcortical deficits may occur exclusively, with infarcts involving the thalamus, basal ganglia, or internal capsule. These may present without any sudden deteriorations, rather manifesting as impaired attention and poor executive function. It is important to differentiate this syndrome from other causes of subcortical dementia and it may coexist with other dementias (for example, AD). Small vessel disease is associated with a syndrome of gait apraxia, urinary incontinence, and pseudobulbar palsy. Urinary and gait disturbances typically occur relatively early in the disease course and sometimes before there are overt signs of cognitive impairment. This pattern of disease is associated with brain imaging findings of lacunes in the deep grey matter nuclei with associated ischaemic demyelination histopathologically (known as Binswanger's disease). A combination of cortical and subcortical pathology is not uncommonly seen leading to a mixture of cognitive impairments.

\section{Dementia with Lewy bodies (DLB)}

Lewy bodies are neuronal inclusions composed of abnormally phosphorylated neurofilament proteins aggregated with ubiquitin and $\alpha$-synuclein that are deposited in brainstem nuclei, paralimbic, and neocortical areas. The clinical phenotype often involves visual hallucinations, parkinsonism, and fluctuating attention and alertness with intervals of lucidity. The visual hallucinations are typically well formed and detailed. Hallucinations occur in other modalities not uncommonly and delusions also are a feature. The cognitive profile reflects a combination of cortical and subcortical disease (table 5). There is cognitive slowing with impairment of frontal executive functions and attention. In addition there are pronounced visuospatial and memory problems implicating parieto-occipital regions. The presence of aphasia, agnosia, and apraxia may lead to confusion with AD. Along with the cognitive effects, DLB is associated with repeated falls and episodes of transient loss of consciousness. 

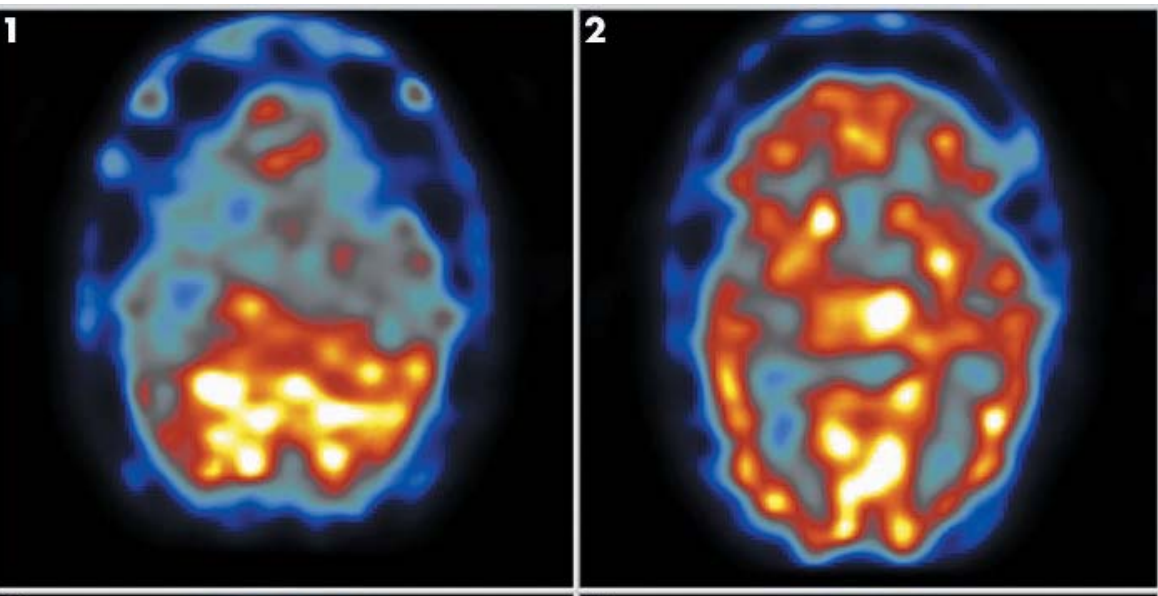

Figure 6 Bilateral frontotemporal hypoperfusion on SPECT in

frontotemporal dementia.
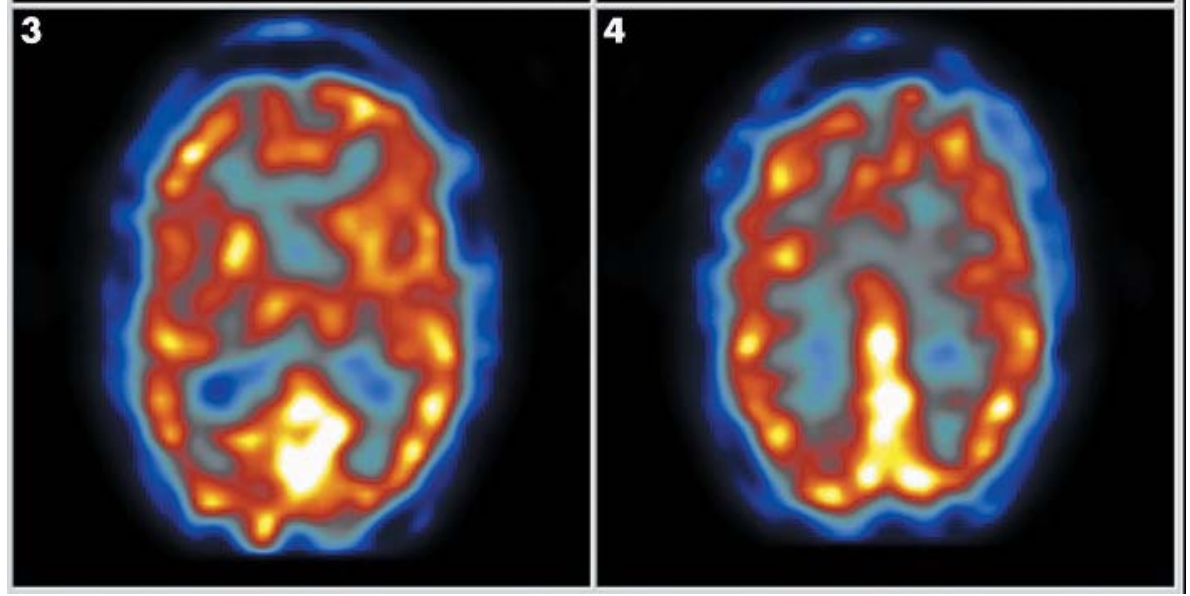

These cognitive impairments may develop before or after parkinsonism symptoms and signs including akinesia, rigidity, and tremor.

\section{Corticobasal degeneration}

Corticobasal degeneration (CBD) usually presents with an asymmetric akinetic-rigid syndrome, progressing to death within 4-6 years. Ideomotor limb apraxia is often observed and there are associated visuospatial and constructional difficulties. The alien hand sign (spontaneous, coordinated hand movements outside of the patient's control) may develop in one limb. This may be associated with cortical sensory loss, dysarthria, ataxia, chorea, pyramidal signs, dysarthria and/or buccofacial apraxia. Dyscalculia and a nonfluent aphasia may be observed and frontal dysfunction may also occur. Memory impairment is typically less pronounced than that observed in $\mathrm{AD}$.

\section{REVERSIBLE DEMENTIAS AND HOW TO IDENTIFY THEM}

Reversible dementias represent a minority of dementias but the importance of identifying them is obvious. Clinicians should have an understanding of what may differentiate reversible dementias from the progressive, largely untreatable neurodegenerative conditions. Some "treatable" dementias may not be cured, but the disease course may be modified by

Table 5 Cognitive features of Alzheimer's disease (AD), frontotemporal dementia (FTD), and dementia with Lewy Bodies (DLB)

\begin{tabular}{|c|c|c|c|}
\hline Cognitive domain & AD & FTD & DLB \\
\hline $\begin{array}{l}\text { Anterograde episodic memory (e.g. } \\
\text { address recall) }\end{array}$ & Typically impaired & Preserved early in the disease & $\begin{array}{l}\text { Recognition better than recall. } \\
\text { Forgetful }\end{array}$ \\
\hline Semantic memory & Less affected than anterograde memory & $\begin{array}{l}\text { Impaired in semantic dementia } \\
\text { (progressive non-fluent aphasia) }\end{array}$ & \\
\hline Verbal fluency (e.g. naming lists) & $\begin{array}{l}\text { Category fluency typical more impaired } \\
\text { than letter based verbal fluency }\end{array}$ & $\begin{array}{l}\text { Decreased verbal fluency with } \\
\text { perseveration }\end{array}$ & Reduced output \\
\hline $\begin{array}{l}\text { Frontal "executive" function } \\
\text { Working memory (e.q. diqit span) }\end{array}$ & $\begin{array}{l}\text { Preserved in early stages } \\
\text { Preserved }\end{array}$ & $\begin{array}{l}\text { Impaired } \\
\text { Impaired (poor concentration) }\end{array}$ & Impaired from onset \\
\hline Visuospatial function & Often preserved in early stages & Preserved in early stages & $\begin{array}{l}\text { Often impaired, hallucinations are } \\
\text { common }\end{array}$ \\
\hline Social interaction & Strikingly preserved & $\begin{array}{l}\text { Affected early, stereotyped, } \\
\text { perseverative behaviours may exist }\end{array}$ & $\begin{array}{l}\text { Fluctuations in function frequently } \\
\text { described, associated with delusions. } \\
\text { Depression common }\end{array}$ \\
\hline
\end{tabular}




\begin{tabular}{l} 
Table 6 Illnesses associated with a potentially treatable \\
dementia \\
\hline Depressive pseudodementia \\
Space occupying lesions \\
Benign tumours, especially subfrontal meningiomas \\
Subdural haematoma \\
Hydrocephalus \\
Deficiency states \\
Vitamins B12, B1 (Wernicke-Korsakoff), B6 \\
Niacin (Pelllagra) \\
Endocrine disease and metabolic disorders \\
Hypothyroidism \\
Chronic hypocalcaemia \\
Recurrent hypoglycaemia \\
Cushing's disease \\
Addison's disease \\
Uraemia \\
Hepatic encephalopathy \\
Hashimoto's encephalopathy \\
Infections \\
AlDS dementia complex \\
Lyme disease \\
Tuberculosis (TB) \\
Syphilis \\
Inflammatory and vasculitides \\
Systemic lupus erythematosis \\
Giant cell arteritis \\
Polyarteritis nodosa \\
Behçet's disease \\
Neurosarcoidosis \\
Alcoholic dementia \\
Chronic intoxications \\
Heavy metals \\
Drugs \\
Carbon monoxide poisoning \\
Wilson's disease \\
Whipple's disease \\
Limbic encephalitis (paraneoplastic or associated with voltage gated \\
potassium channel antibodies) \\
\hline
\end{tabular}

addressing the underlying cause (for example, vascular disease and modification of risk factors). Causes of dementia amenable to treatment are outlined in table 6. Examples of non-neurological clinical features associated with specific, potentially treatable causes of dementia are shown in table 7.

Imaging can contribute to making a positive diagnosis of dementia, such as MRI showing hippocampal atrophy in early AD. It is, however, also of use in excluding some reversible causes of dementia, such as a benign tumour. Claims that clinical prediction rules can allow the clinician to differentiate between, say, early $\mathrm{AD}$ and space occupying lesions have not been borne out by the evidence, and we feel that, at the least, a brain CT is mandatory for investigating the dementing patient.

\section{Benign tumours}

Change in personality may be the only manifestation of a slow growing frontal tumour such as a meningioma. Deeper tumours, around the pituitary or third ventricle, may present solely with cognitive impairment.

\section{Chronic subdural haematoma}

This can present with subacute dementia, often with fluctuations. There may be no obvious history of head trauma, especially in alcoholics or in patients on anticoagulants. It is eminently surgically treatable, and must not be missed.

\section{Normal pressure hydrocephalus}

Traditionally, it was claimed that this disorder should be suspected if the clinical triad of gait apraxia, subcortical dementia, and urinary dysfunction are present. The feet are said to show the "glued to the floor" sign when the patient tries to walk. Imaging shows ventricular dilatation out of proportion to the degree of sulcal enlargement. Subsequent pressure monitoring may show B waves, or a therapeutic trial of lumbar puncture may significantly improve gait, in which case shunting is indicated.

There is, however, current debate as to whether this entity exists. It is claimed that such patients may in fact have $A D$, and the apparent improvement from lumbar puncture may relate to changes in amyloid in cerebrospinal fluid (CSF) as a result of this procedure.

\section{Metabolic and endocrine}

Metabolic disorders tend to produce delirium rather than dementia, but hypocalcaemia and recurrent hypoglycaemia can present with dementia, usually with a movement disorder.

Hypothyroidism must obviously be excluded in any patient with cognitive impairment. Addison's and hypopituitarism may occasionally present with cognitive impairment.

In any young patient with dementia, especially if showing a subcortical pattern with a movement disorder, Wilson's disease must be excluded. This is a disorder of copper metabolism, and treatment becomes less effective if the diagnosis is delayed.

\section{Infections}

HIV may cause a direct infection of the brain, resulting in a subcortical dementia. White matter changes are seen on MRI, and CSF shows a pleocytosis with oligoclonal bands. A more rapidly progressive dementia can occur in AIDS due to opportunistic infections, such as cerebral toxoplasmosis,

Table 7 Examples of non-neurological clinical features associated with potentially treatable causes of dementia

\begin{tabular}{ll}
\hline Clinical sign & Disease \\
\hline Lymphadenopathy & HIV/AIDS, malignancy, \\
& sarcoidosis, tuberculosis (TB) \\
Signs of immunocompromise & HIV/AIDS, malignancy \\
O Herpes simplex ("cold sores") & \\
1 Herpes zoster & \\
2 Oral candida & \\
3 Hairy leucoplakia & \\
4 Kaposi's sarcoma & \\
Hepatomegaly & Malignancy \\
Cachexia & Nutritional deficiency \\
& Alcoholism \\
& Malignancy \\
Hypertension & AIDS \\
Kaiser-Fleischer rings & Vascular disease \\
Uveitis & Wilson's disease \\
Rash & Sarcoid, TB, multiple sclerosis \\
5 Erythema nodosum & \\
6 Vasculitic purpura & Sarcoid, TB \\
7 "Butterfly" rash & Systemic vasculitides \\
8 Erythema chronicum migrans & Systemic lupus erythematosus (SLE) \\
9 "Casel's necklace" & Lyme disease \\
Non-pulsatile, tender temporal & Giant cell arteritis \\
arteries & Addison's disease \\
Hyperpigmentation of skin & Cushing's syndrome \\
Cushingoid appearance & Hypothyroidism \\
Pretibial myxoedema & Hypothyroidism \\
Loss of eyebrows & Behçet's disease \\
Oral/genital ulceration & Lead poisoning \\
Blue gums (a "lead line") & Whipple's disease, SLE, Lyme \\
Arthritis & disease, Behçet's disease \\
& \\
\hline & \\
& \\
& \\
& \\
&
\end{tabular}


cryptococcal meningitis, or progressive multifocal leucoencephalopathy.

Syphilis, although rare, is becoming less uncommon, and should be considered in the dementing patient, especially if accompanied by supportive neurological signs such as Argyll Robertson pupils.

Whipple's disease should be considered if there is oculomasticatory myorhythmia, ocular palsies, or ataxia.

\section{Cerebral vasculitis}

When considering a diagnosis of vasculitis it is important to remember that vasculitis may occur as a primary condition (that is, Wegener's granulomatosis, temporal arteritis, polyarteritis nodosa, Churg-Strauss syndrome, primary angiitis of the nervous system) or as part of a multisystem disorder that may cause a vasculitis (for example, systemic lupus, sarcoid, Behçet's disease, lymphoma, and cryoglobulinaemia). Vasculitis affecting the central nervous system in isolation is rare.

\section{Autoimmune encephalopathies}

Paraneoplastic limbic encephalitis may present with subacute onset cognitive impairment, often with seizures, and is associated with paraneoplastic antibodies. This disorder gives the clinician the opportunity to identify an occult primary cancer at an early stage, with the possibility of early treatment.

Certain other antibodies have been identified in the context of cognitive impairment-for example, thyroid antibodies in Hashimoto's encephalopathy. Whether such antibodies are implicated in the pathogenesis is, however, less clear.

More recently, a voltage gated potassium antibody mediated limbic encephalitis has been described, which appears to respond well to immunosuppression if diagnosed and treated early.

..................

Authors' affiliations

S Cooper, J D W Greene, Southern General Hospital, Institute of Neurological Sciences, Glasgow, UK

\section{REFERENCES}

1 Hodges JR, Greene JDW. Disorders of memory. In: Kennard C, eds. Recent advances in clinical neurology 8. Churchill Livingstone, 1995.

2 Greene JDW, Hodges JR. The dementias. In: Berriers GE, Hodges JR, eds. Memory disorders in neuropsychiatric practice. Cambridge: Cambridge University Press, 2000:122-61.

3 Neary D, Snowdon JS. Sorting out the dementias. Pract Neurol 2002;2:328-39.

4 Sampson EL, Warren JD, Rossor MN. Young onset dementia. Postgrad Med 2003;80:125-39.

5 Kipps CM, Hodges JR. Cognitive assessment for clinicians. J Neurol Neurosurg Psychiatry 2005;76(suppl I):i22-30.

6 Folstein MF, Folstein SE, McHugh PR. "Mini mental state". A practical method for grading the cognitive state of patients for the clinician. J Psychiatr Res 1975; 12:189-98.

7 Mathuranath PS, Xuereb JH, Bak T, et al. Corticobasal degeneration of frontotemporal dementia? A report of two cases and a review of the literature. J Neurol Neurosurg Psychiatry 2000;68:304-12.

8 Neary D, Snowden JS. Sorting out the dementias. Practical Neurology 2002;2:328-39. 